\title{
Measurements of Magnetic Properties of Electromagnetic Actuator in High-Temperature Environment
}

\author{
M. Noh ${ }^{1 *}$, M. J. Gi' ${ }^{1}$, D. Kim ${ }^{1}$, Y.-W. Park ${ }^{1}$, J. Lee ${ }^{2}$ and J. Kim² \\ ${ }^{1}$ Chungnam National University, 99 Daehak-ro, Yuseong-gu, Daejeon 305-764, Korea \\ ${ }^{2}$ Korea Atomic Energy Res. Inst., Daedeok-daero 989-111, Yuseong, Daejeon 305-353, Korea
}

(Received 2 January 2015, Received in final form 16 March 2015, Accepted 16 March 2015)

\begin{abstract}
Electromagnetic actuators are versatile and able to meet demanding requirements, such as operation in very low or very high temperatures. When the actuator is used in a high-temperature environment up to $500^{\circ} \mathrm{C}$, we need to know how the force-producing capability of the actuator is affected by the operating temperature. Specifically, it is necessary to know the temperature-dependence of magnetic properties that determine the mechanical forces. In this paper, we measure the changes in magnetic properties of SUS410 material in hightemperature environment. We also devise a novel signal processing technique to remove the integration drift. At the field strength of $18,000 \mathrm{~A} / \mathrm{m}$, we found that the flux density at $500^{\circ} \mathrm{C}$ is decreased by $26 \%$, compared to the result at room temperature. Therefore, the actuator must be sized appropriately, if it is to operate in hightemperature settings.
\end{abstract}

Keywords : electromagnetic actuator, high-temperature environment, magnetic property, magnetic stainless steel

\section{Introduction}

An electromagnetic actuator is a device that produces mechanical forces by converting electric energy by means of the change in magnetic field. Electromagnetic actuators are able to impart mechanical forces without contact, and thus highly efficient and typically long-lasting compared to traditional mechanical counterparts such as gears, cams, or links. They have found wide industrial applications in motion generation and control including electric machines, solenoids, and magnetic levitation systems. They are also able to meet requirements in demanding applications operating in cryogenic, vacuum, or hightemperature environments.

When electromagnetic actuators are to be used in hightemperature environments, it is crucial to know how the force-producing capability of the actuator is affected by the temperature it is operated in. Specifically, it is necessary to know the temperature-dependence of magnetic properties that determine the mechanical forces. There have been several studies related to this topic in efforts to use electromagnetic actuators in high-temperature environ-

CThe Korean Magnetics Society. All rights reserved.

*Corresponding author: Tel: +82-42-821-6877

Fax: +82-42-823-4919, e-mail: mnoh@cnu.ac.kr ments. For example, Xu et al. [1] measured the maximum flux density of AFK502 (Fe 50\% and Co 50\%) material up to around $1000^{\circ} \mathrm{C}$ and found that the decrease in the maximum flux density is less than $30 \%$ up to $850^{\circ} \mathrm{C}$. Kondoleon and Kelleher [2] tested mechanical and magnetic properties of several materials at room temperature and at $550^{\circ} \mathrm{C}$. They obtained similar results for Hiperco 50, which is identical to AFK502 in composition.

In some applications, it is not possible to use materials with significant proportion of cobalt for economic reasons or due to susceptibility to radiation. Hossain [3] tested AISI 1010 (low carbon steel) as well as Hiperco 50, and found that AISI 1010 can be used up to $985^{\circ} \mathrm{F}\left(530^{\circ} \mathrm{C}\right)$ with the $24 \%$ sacrifice in the maximum flux density. If the actuator is operating in humid or submersed applications such as in nuclear reactors, it is advisable to use rust-free materials. Although magnetic stainless steels are not very good magnetic materials, they are excellent in durability and robust in high-temperature settings. In literature, however, little can be found on the magnetic properties of stainless steels, let alone their high-temperature characteristics. Ishida et al. [4] provided test results on the change in the magnetization characteristics of SUS410 at room temperature and at $300^{\circ} \mathrm{C}$. However, they did not present any data in temperatures up to $500^{\circ} \mathrm{C}$. 
The measurements of magnetic properties at high temperatures require a setting that can accommodate the high temperature environment enclosing a specimen. A ring test is suitable for this purpose, where a primary coil magnetizes a ring core and a secondary coil picks up the induced voltage and integrates it to measure the magnetic flux. This approach, however, calls for a method to remove the integration drift resulting from the electrical offset present in the induced voltage signals. Typically, a very large amount of measurements need to be collected to estimate and compensate the drift.

In this paper, we present the experimental results regarding magnetic properties of SUS410 in an environment where the temperature is up to $500^{\circ} \mathrm{C}$. In order to remove the integration drift from the measurements, we also develop a novel method of estimating the drift using the modest size of data. The section immediately following this describes how the tests have been carried out at various temperatures. We also explains how the measurements are processed in order to obtain the magnetic properties. The section on results and discussions shows the measured magnetic data and presents discussions on the results. The paper ends with some conclusions.

\section{Measurements of Temperature- Dependent Magnetic Properties}

\subsection{Measurement Setup}

Since we are interested in the temperature-dependent magnetic properties of the core material, we need a test setup that measures the magnetic property while maintaining a constant-temperature environment. The commonly-used vibrating sample magnetometer (VSM) is excellent equipment to measure magnetic properties for wide temperature range, but difficult to measure hysteresis loop of soft magnetic materials due to the demagnetizing effect. In this research, we select the Rowland ring test [5, 6]. A ring made of the material to be tested is wound with two sets of coils: primary and secondary.

When an alternating current is applied to the primary coil, the inside of the ring core is magnetized with the magnetic field strength of

$$
H_{c}=\frac{N_{p} I_{p}}{l_{c}},
$$

where $N_{p}$ is the number of primary coil turns, $I_{p}$ is the primary current and $l_{c}$ the mean length of the core. The resulting magnetic flux is time-varying because of the alternating nature of the primary current. A voltage is induced in the secondary coil, which can be expressed as

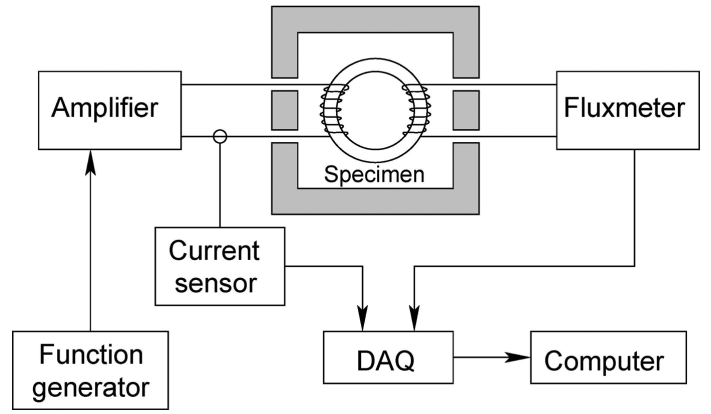

Fig. 1. Schematic of test setup.

$$
e_{\text {ind }}=-N_{s} \frac{d \phi}{d t},
$$

where $N_{s}$ is the number of secondary coil turns. Therefore, the flux density inside the coil can be obtained as

$$
B_{c}=-\frac{1}{N_{p} A_{c}} \int e_{\text {ind }} d t .
$$

Here, $A_{c}$ is the cross-sectional area of the core.

The schematic of the measurement setup is shown in Fig. 1. A function generator provides a sinusoidal input with the frequency of $0.1 \mathrm{~Hz}$ to a current amplifier (JSP180-20, Copley Controls, USA) which then generates the primary current with the same frequency. A flux meter (MF-100, Walker Scientific, USA) measures the induced voltage in the secondary coil and converts it into the flux density by performing the integration. A current sensor measures the primary current. The current signals and flux density measurements are converted into digital data and recorded to a computer for signal processing. The sampling rate of the data acquisition (DAQ) is $1 \mathrm{kHz}$ and four cycles of data are recorded.

The ring specimen is located inside an oven which can maintain the temperature of up to $1000^{\circ} \mathrm{C}$. The measurement sequence is as follows. The magnetic property of the core material is measured at room temperature. Then, the temperature is elevated to $50^{\circ} \mathrm{C}$ for thirty minutes. Once the oven temperature reaches $50^{\circ} \mathrm{C}$, we wait another thirty minutes for thermal equilibrium before starting the magnetization measurement routine. This process is repeated for every $50^{\circ} \mathrm{C}$ interval up to $500^{\circ} \mathrm{C}$.

\subsection{Signal Processing}

When performing the integration in eq. (3), it is important to eliminate the integration drift due to unavoidable electrical offsets that are present in the induced voltage signal. Commercially available fluxmeters are equipped with a nulling function which eliminates the offset through either software or hardware means. However, even a tiny offset that remains after nulling can be problematic 
because the drift accumulates over time. Therefore, it is necessary to remove the integration drift after the integration, while keeping the offset small so that the integrated signals stay within the measurement range.

For a robust drift removal, however, the signals must first pass through low-pass filters that remove highfrequency electrical noises. The low-pass filters can be digitally implemented by a conventional infinite-impulse response (IIR) filters that can be expressed as

$$
y_{n}=\sum_{k=1}^{m} a_{k} x_{n-k+1}+\sum_{k=1}^{m-1} b_{k} y_{n-k}
$$

The IIR filter of (4) is causal as the present filtered signal $y_{n}$ depends only on the present and past values of the input signal $x_{k}$ and the past values of the filtered signal $y_{k}$. The filter depth $m$ determines the order of the filter. This type of IIR filter would introduce phase delays, resulting in distortions in measurements.

In order to avoid the phase delay, a non-causal digital filter can be used. A non-causal filter looks ahead and utilizes the future values of the input. The filter that is used in this paper is a zero-phase forward-backward filter [7] which completely eliminates the phase delay. A noncausal low-pass filter with the cut-off frequency of $50 \mathrm{~Hz}$ is designed and digitally applied to the current and the flux density signals. Fig. 2 shows the filtered flux density measurements, which clearly displays the integration drift.

A usual method of drift cancellation is detrending that is to subtract either the mean value or the linear fit of the data. This method does not work well for the flux density measurement, because the acquired data length is not exactly multiples of the period. The mean value or the linear fit of the data is very sensitive to the end mismatch (either slightly shorter or longer than a period). In order to

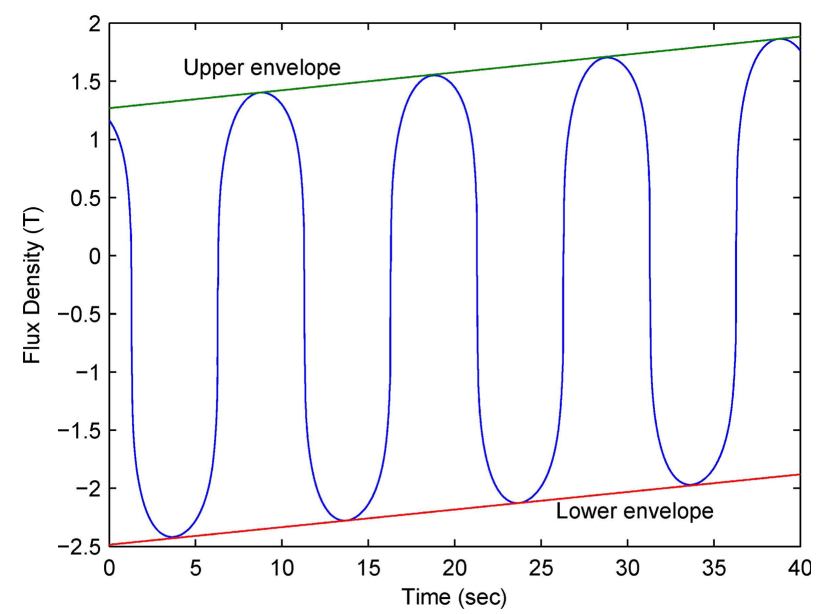

Fig. 2. (Color online) The raw output of the fluxmeter and the identified evenlopes. solve this problem, we have devised a novel method of estimating the envelopes and using these envelopes to remove the drift.

Assuming that the drift is linear with respect to time because of a constant offset in the integrator, the ridges and troughs of the flux density curve would be bounded by linear envelopes. The condition that these lines are to be envelopes would be to touch either ridges or troughs and never to cross into the flux density curve. This condition can be formulated into an optimization problem of minimizing the distance between the envelope and the curve. Fig. 2 shows the upper and lower envelopes identified by this method. The drift can be eliminated by subtracting the average of the two envelopes from the flux density curve.

The final step in signal processing is obtaining the magnetization curve from the hysteresis curve constructed by the corrected flux density values with respect to the magnetizing intensity of eq. (1). Although the hysteresis curve itself can be used for analyzing and designing an electromagnetic actuator, a single-valued magnetization curve would be more useful for finite-element analyses (FEA) or magnetic circuit analyses. In this paper, it is assumed that the magnetization curve is the median curve dissecting the hysteresis curve as shown in Fig. 3.

\section{Results and Discussions}

A toroidal ring made of SUS410 is manufactured, the dimension of which is as follows: inner diameter $30 \mathrm{~mm}$, outer diameter $40 \mathrm{~mm}$, and thickness $2.1 \mathrm{~mm}$. The primary coil has 300 turns, while the secondary coil-turns is 20 . The magnetic property of the specimen is measured at room temperature and from $50^{\circ} \mathrm{C}$ to $500^{\circ} \mathrm{C}$ at $50^{\circ} \mathrm{C}$

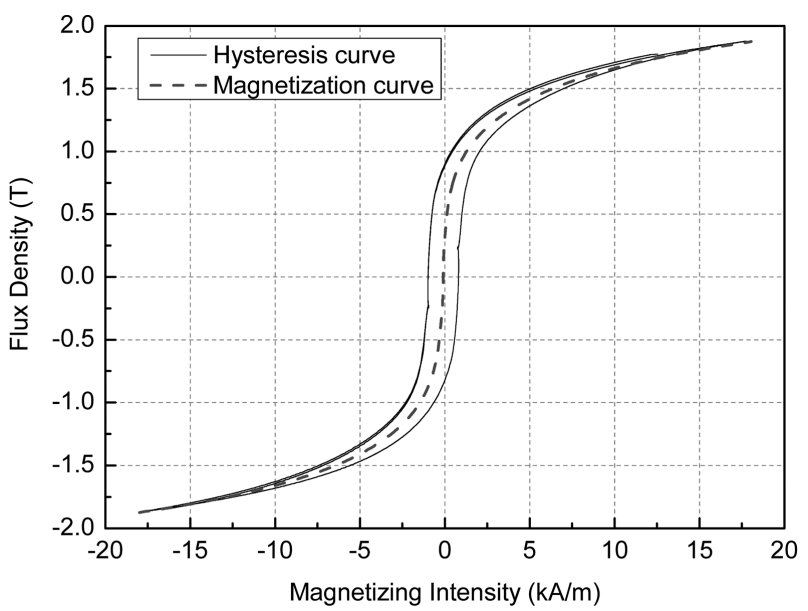

Fig. 3. Hysteresis curve obtained from the measurements and the magnetization curve. 


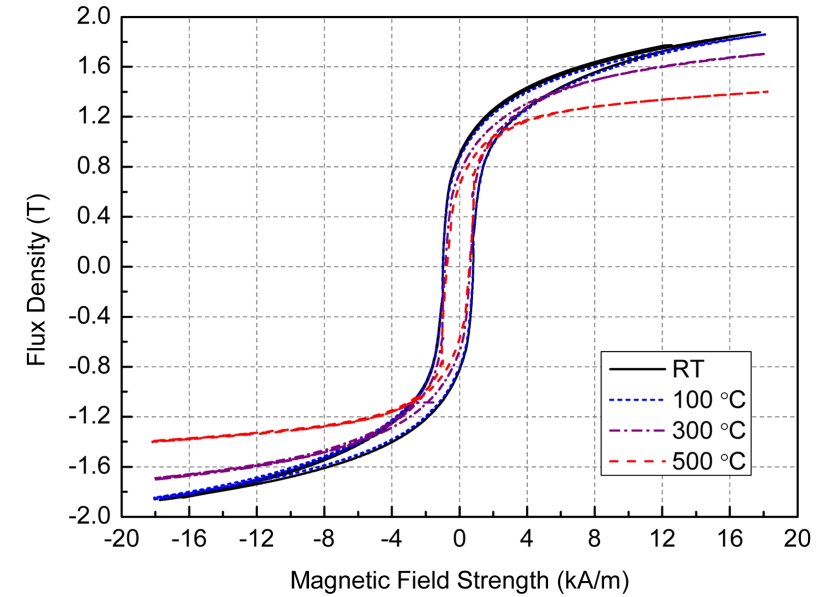

Fig. 4. (Color online) Hysteresis curves obtained at the room temperature (RT), $100^{\circ} \mathrm{C}, 300^{\circ} \mathrm{C}$, and $500^{\circ} \mathrm{C}$. Other measurements are not shown for clarity.

interval: total of 11 data sets. Each data set is processed as described previously.

Fig. 4 shows the hysteresis curves measured at room temperature, $100^{\circ} \mathrm{C}, 300^{\circ} \mathrm{C}$, and $500^{\circ} \mathrm{C}$. The rest of the data sets are not displayed for clarity. The results indicate that the magnetic property changes little from room temperature up to $100^{\circ} \mathrm{C}$. Even at $300^{\circ} \mathrm{C}$, the variation in magnetic property is minor. However, there is a substantial change in the high field region when the temperature reaches $500^{\circ} \mathrm{C}$. These changes are more evident in the median B-H curves as demonstrated in Fig. 5. The median B-H curves are obtained from the center line dissecting the hysteresis curves and can be considered as an estimate of anhysteretic curve [8]. At the magnetic field strength of

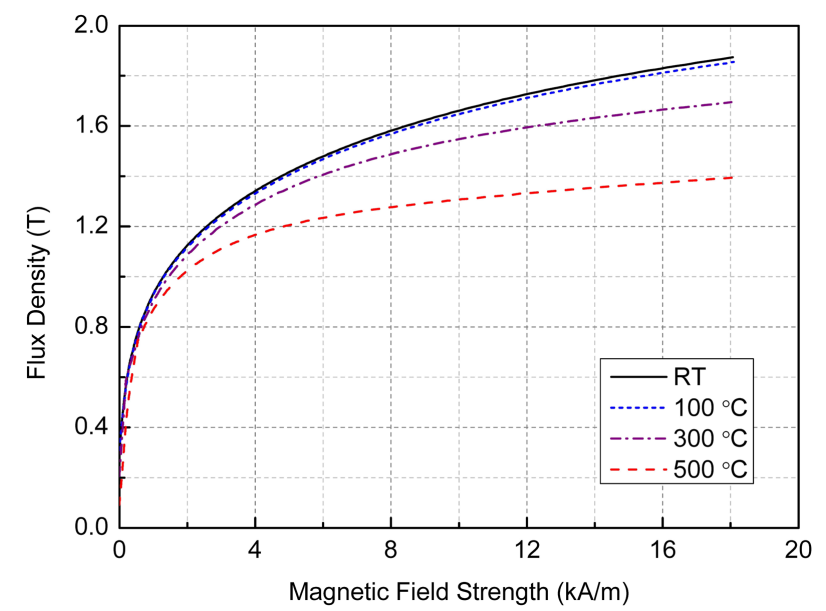

Fig. 5. (Color online) Median $\mathrm{B}-\mathrm{H}$ curves obtained at the room temperature $(\mathrm{RT}), 100^{\circ} \mathrm{C}, 300^{\circ} \mathrm{C}$, and $500^{\circ} \mathrm{C}$. Other results are not shown for clarity.

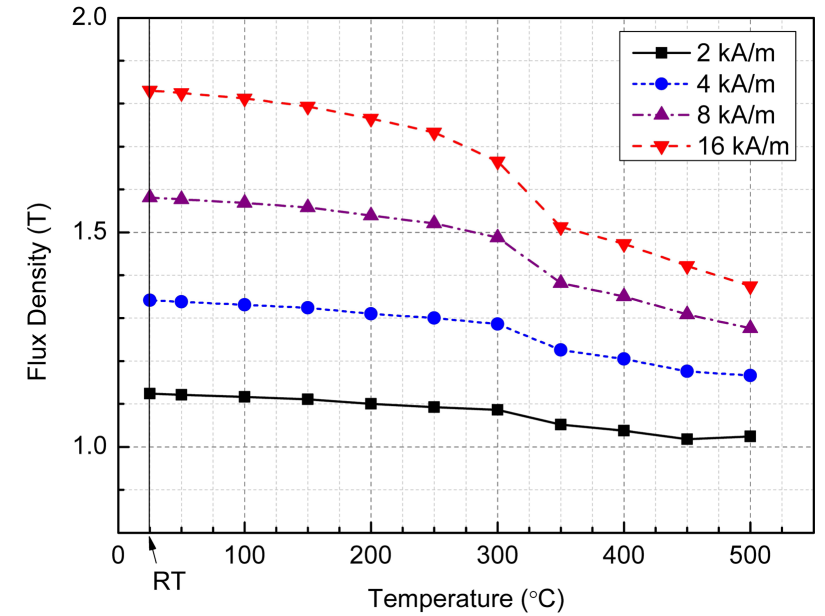

Fig. 6. (Color online) The variations of the magnetic flux density with respect to the temperature at several levels of magnetic field strength.

$18,000 \mathrm{~A} / \mathrm{m}(226 \mathrm{Oe})$, the flux density drops from $1.87 \mathrm{~T}$ at room temperature to $1.39 \mathrm{~T}$ at $500^{\circ} \mathrm{C}(26 \%$ decrease). This would result in $45 \%$ reduction in the force producing capability of the actuator since the force is proportional to the square of the flux density, although it would take very high field until this reduction matters. At a more likely strength of $4000 \mathrm{~A} / \mathrm{m}$ (50 Oe), the flux density is reduced from $1.34 \mathrm{~T}$ at room temperature to $1.17 \mathrm{~T}$ at $500^{\circ} \mathrm{C}(13 \%$ decrease), resulting in $26 \%$ reduction in the actuator force. When designing an actuator operating in hightemperature environment, it is therefore necessary to oversize the actuator. The amount of oversizing depends on how much the actuator is required in the saturation region.

Fig. 6 summarizes the changes in the flux densities due to temperatures at various levels of magnetic field strength. At low strength, the flux density change is minor, which progressively worsens as the field strength increases.

\section{Conclusion}

In this paper, we measured the magnetic properties of a magnetic stainless steel, SUS410 while the surrounding temperature was varied up to $500^{\circ} \mathrm{C}$. In order to remove the integration drift, inevitable artifact when integrating the induced voltage, we developed a method of estimating the drift using an optimization technique. At high levels of magnetic field strength, we found that the flux density decreases much $(26 \%$ at $18,000 \mathrm{~A} / \mathrm{m})$, which results in substantial loss of force producing capability of an electromagnetic actuator. Thus, the actuator must be sized appropriately if it is to operate in high-temperature settings. 


\section{Acknowledgements}

This work was supported by the National Research Foundation of Korea (NRF) grant funded by Korea Government (MSIP) (No. 2012M2A8A4026290).

\section{References}

[1] L. Xu, L. Wang, and G. Schweitzer, Proc. Seventh Int. Symp. Mag. Brg. (ISMB7) 117 (2000).

[2] A. S. Kondoleon and W. P. Kelleher, Proc. Seventh Int. Symp. Mag. Brg. (ISMB7) 111 (2000).
[3] M. A. Hossain, Master Thesis, Texas A\&M University, U.S.A. (2006).

[4] T. Ishida, S. Imayoshi, T. Yoritsune, H. Nunokawa, M. Ochiai, and Y. Ishizaka, J. Nucl. Sci. Technol. 38, 557 (2001).

[5] D. Halliday, R. Resnick, and J. Walker, Fundamentals of Physics, John Wiley \& Sons, New York (2001) p. 755.

[6] ASTM A 773, American Society of Testing and Materials (2009).

[7] A. V. Oppenheim and R. W. Schafer, Discrete-Time Signal Processing, Prentice-Hall, New Jersey (1989) p. 284.

[8] D. Jiles, Introduction to Magnetism and Magnetic Materials, Chapman \& Hall, London (1998) p. 115. 